\title{
High-flow oxygen therapy in cluster headache patients has no significant effect on nociception specific blink reflex parameters: a pilot study
}

\author{
D. Y. P. Haane ${ }^{1,2^{*}}$, A. Plaum ${ }^{1,3}$, P. J. Koehler ${ }^{1}$ and M. P. W. A. Houben ${ }^{1}$
}

\begin{abstract}
Background: The exact pathophysiology of cluster headache is unclear. We examined the influence of interneurons on the trigemino-facial reflex arch and the effect of oxygen, by using the nociception specific blink reflex parameters.

Findings: There is no significant effect of oxygen, immediately and over time, on the nociception specific blink reflex parameters in ten male patients during the active phase of cluster headache, outside attacks. Also, there is no significant difference between the symptomatic and asymptomatic side. None of the subjects experienced a cluster headache attack during study participation. We therefore present the collected data as reference values of nociception specific trigeminal stimulation and the effect of oxygen on nociception specific blink reflex parameters.

Conclusion: The nociception specific blink reflex seems not a suitable instrument for exploring the pathophysiology of cluster headache.
\end{abstract}

Keywords: Cluster headache, Nociception specific blink reflex, Oxygen therapy, Normal values

\section{Introduction}

The exact pathophysiology of cluster headache $(\mathrm{CH})$ is unclear. Previous studies have shown that $100 \%$ oxygen $\left(\mathrm{O}_{2}\right)$ therapy is a notable $\mathrm{CH}$ attack reliever [1]. Exactly how oxygen exerts its pain reducing effect in patients with $\mathrm{CH}$ is uncertain, but it is shown to directly or indirectly cause vasoconstriction. Indirect vasoconstriction can be the result of a possible action on the parasympathetic outflow from the superior salivatory nucleus, as is shown in rats [2].

The blink reflex (BR) is a brainstem reflex, elicited through stimulation of the supraorbital nerve, derived from the first branch of the trigeminal nerve, resulting in a bilateral blink reaction of the eyelids through the

\footnotetext{
* Correspondence: dhaane@hotmail.com

'Department of Neurology and Clinical Neurophysiology, Zuyderland Medical Center Heerlen, P.O. Box 5500, 6130 MB Sittard, The Netherlands

${ }^{2}$ Current address: Department of Neurology, Mariaziekenhuis Noord-Limburg, Overpelt, Belgium

Full list of author information is available at the end of the article
}

facial nerve. The BR is composed of an early pontine response (R1), and a late medullary response (R2) [3]. R1 is oligosynaptic, ipsilateral and not clinically visible, whereas R2 is polysynaptic, bilateral and clinically observable [4]. A nociception specific blink reflex (nBR) can be elicited by transcutaneously selectively stimulating superficial nociceptive A-delta fibers of the supraorbital nerve with a concentric planar stimulating electrode. The response consists of only a bilateral R2. Using the $n B R$, the function of the afferent trigeminal and efferent facial nerves and their central connections can be assessed [3].

We wanted to examine the influence of interneurons on the trigemino-facial reflex arch and the effect of high-flow (12 liter/minute $(\mathrm{L} / \mathrm{min})) \mathrm{O}_{2}$ by using the $\mathrm{nBR}$ and its parameters. However, none of the subjects experienced a $\mathrm{CH}$ attack during study participation, despite the fact that all of the subjects were in a cluster period at the time. This was possibly due to a preventive effect of nociception specific trigeminal stimulation on $\mathrm{CH}$

\section{Springer}


attacks [5]. We therefore present the data as reference values of the nBR parameters in patients in a cluster period outside a $\mathrm{CH}$ attack and the effect of high-flow $\mathrm{O}_{2}$ inhalation.

\section{Methods}

Information concerning study population, in- and exclusion, equipment and questionnaires was already described in a previous publication [5]. The study was approved by the local ethics committee. All patients gave written informed consent. The study terminated early because none of the patients experienced a $\mathrm{CH}$ attack during clinical study time. One patient was excluded because the diagnosis of $\mathrm{CH}$ was questioned following study participation. $\mathrm{CH}$ patients were not compared to healthy controls; the baseline measurement was considered a control.

We elicited nBRs in eleven patients using Synergy EMG equipment (Natus Neurology). For stimulation we used a concentric planar electrode with central cathode and external anode ring ( $\mathrm{K} 2$ concentric ring stimulating electrode, $1.5 \mathrm{~mm}$; Inomed, Emmendingen, Germany). Disposable silver/silver chloride electrodes were placed over the orbicularis oculi muscles, just lateral of the mid-pupillary line (active) and near the lateral canthus (reference). The ground electrode was placed on the chin. The supraorbital nerve was stimulated ten millimeter cranial of the supraorbital notch with a 200 pulse per second (pps) train of three $0.5 \mathrm{~ms}$ pulses. The current intensity was increased stepwise by $0.3 \mathrm{~mA}$, with regard to the tolerance limit of the patient, up to $20 \%$ above the level that acquired stable R2 responses to assure supramaximal stimulation, with a maximum of $2.1 \mathrm{~mA}$ (once $2.4 \mathrm{~mA}$ ). The stimuli were delivered at unpredictable intervals of at least $15 \mathrm{~s}$ to minimize habituation. Both the symptomatic and the asymptomatic side were stimulated until we had obtained four blink reflexes on each side (here referred to as one measurement). In each subject, the $\mathrm{R} 2$ responses were elicited at at least the five time points: before $\mathrm{O}_{2}$ inhalation, during $\mathrm{O}_{2}$ inhalation and every two hours thereafter up until six hours after $\mathrm{O}_{2}$ inhalation. It was originally planned to continue until a spontaneous $\mathrm{CH}$ attack occurred, but this did not happen.

We analyzed the measurements before, during and six hours after $\mathrm{O}_{2}$ inhalation. All responses were evaluated by two researchers ( $\mathrm{DH}$ and $\mathrm{MH})$. For each stimulation site and time we calculated the shortest latency, amplitude, duration and area of the R2 response using Synergy Reader version 20.1.0.100 (Natus Neurology).

\section{Statistical analysis}

We performed the analyses using IBM SPSS statistics version 21. Variables were tested for normal distribution
(Shapiro-Wilk). We calculated mean with standard deviation or median with interquartile range as appropriate. Differences of mean were tested with a paired samples t-test. Differences of median were tested using Wilcoxon signed-rank test. Significance levels were adjusted for multiple testing by Bonferroni correction $(p<0.0025)$.

\section{Results}

Ten $\mathrm{CH}$ patients were included. All $\mathrm{CH}$ patients were men. Mean age was 45.7 (range 24-69). Mean BMI was 24.0 (range 20.5-36.0). Three patients had episodic $\mathrm{CH}$, five patients had chronic $\mathrm{CH}$ and two patients were in their first cluster. Six patients experienced attacks on the left side, four on the right. Eight patients were current smokers.

Table 1 shows the nBR parameters of the symptomatic and asymptomatic side after both ipsilateral and contralateral stimulation, and before and during $\mathrm{O}_{2}$ inhalation $(n=10)$. There is no significant difference in the nBR parameters before and during $\mathrm{O}_{2}$ inhalation. There were also no differences in baseline parameters when the symptomatic side was compared to the asymptomatic side. We then studied the difference between the measurements before $\mathrm{O}_{2}$ inhalation and six hours after $\mathrm{O}_{2}$ inhalation $(n=9$; the measurement in one subject was rejected because it was impossible to elicit R2 responses after six hours). This difference was not significant either and we considered the values six hours after $\mathrm{O}_{2}$ inhalation as baseline again.

\section{Discussion}

In this study on the pathophysiology of $\mathrm{CH}$ using twohourly transcutaneous stimulation sequences on the supraorbital nerves to elicit the $\mathrm{nBR}$, none of the included patients did experience a $\mathrm{CH}$ attack during study participation, This may be an important serendipitous discovery for future prophylactic treatment studies, which we have discussed before [5].

Based on the nBR parameters there is no significant effect of $\mathrm{O}_{2}$, immediately and over time. There is also no significant difference between the symptomatic and asymptomatic side of the nBR parameters during the active phase of $\mathrm{CH}$, but outside $\mathrm{CH}$ attacks. The stringent correction for multiple testing poses a risk for false negative results. Using no correction, however, none of the results (except for the 'ipsilateral shortest R2 latency symptomatic side during $\mathrm{O}_{2}$ administration' and 'contralateral area asymptomatic side after six hours') would have been significant.

It would be interesting to observe what will happen at brainstem level during $\mathrm{CH}$ attacks in humans. However, if noninvasive nociception specific supraorbital nerve stimulation (SNS) indeed is confirmed to act in a 
Table 1 Nociception specific blink reflex variables at baseline and during high flow oxygen inhalation (12 L/min)

\begin{tabular}{|c|c|c|c|}
\hline \multirow[t]{2}{*}{ Nociception specific blink reflex variable } & \multirow{2}{*}{$\begin{array}{l}\text { Baseline } \\
\text { Mean (SD) }\end{array}$} & \multirow{2}{*}{$\begin{array}{l}\text { During high flow } \\
\text { oxygen inhalation } \\
\text { Mean (SD) }\end{array}$} & \multirow[b]{2}{*}{$p$-value } \\
\hline & & & \\
\hline \multicolumn{4}{|l|}{ R2 latency, symptomatic side (ms) } \\
\hline Ipsilateral stimulation & $44.95(5.65)$ & $47.09(6.29)$ & 0.225 \\
\hline Contralateral stimulation & $48.75(6.53)$ & $50.56(6.03)$ & 0.392 \\
\hline \multicolumn{4}{|l|}{ Shortest R2 latency, symptomatic side (ms) } \\
\hline Ipsilateral stimulation & $39.05(6.55)$ & $43.37(6.19)$ & 0.025 \\
\hline Contralateral stimulation & $44.12(6.69)$ & $44.65(4.16)$ & 0.789 \\
\hline \multicolumn{4}{|l|}{ R2 amplitude, symptomatic side $(\mathrm{mV})$} \\
\hline Ipsilateral stimulation & $0.27(0.10)$ & $0.25(0.08)$ & 0.387 \\
\hline Contralateral stimulation & $0.19(0.07)$ & $0.17(0.07)$ & 0.235 \\
\hline \multicolumn{4}{|l|}{ R2 duration, symptomatic side (ms) } \\
\hline Ipsilateral stimulation & $52.63(14.39)$ & $50.36(13.65)$ & 0.263 \\
\hline Contralateral stimulation & $47.74(19.17)$ & $45.74(14.27)$ & 0.381 \\
\hline \multicolumn{4}{|l|}{ R2 area, symptomatic side (mVms) } \\
\hline Ipsilateral stimulation & $2.28(1.03)$ & $2.04(0.94)$ & 0.202 \\
\hline Contralateral stimulation & $1.57(0.78)$ & $1.25(0.47)$ & 0.132 \\
\hline \multicolumn{4}{|l|}{ R2 latency, asymptomatic side (ms) } \\
\hline Ipsilateral stimulation & $47.62(10.60)$ & $46.01(8.96)$ & 0.223 \\
\hline Contralateral stimulation & $49.93(9.44)$ & $48.85(7.51)$ & 0.418 \\
\hline \multicolumn{4}{|l|}{ Shortest R2 latency, asymptomatic side (ms) } \\
\hline Ipsilateral stimulation & $42.92(10.73)$ & $40.18(9.40)$ & 0.086 \\
\hline Contralateral stimulation & $44.17(10.21)$ & $42.58(8.51)$ & 0.484 \\
\hline \multicolumn{4}{|l|}{ R2 amplitude, asymptomatic side ( $\mathrm{mV}$ ) } \\
\hline Ipsilateral stimulation & $0.32(0.15)$ & $0.27(0.11)$ & 0.100 \\
\hline Contralateral stimulation & $0.19(0.09)$ & $0.16(0.09)$ & 0.174 \\
\hline \multicolumn{4}{|l|}{ R2 duration, asymptomatic side (ms) } \\
\hline Ipsilateral stimulation & $49.29(19.30)$ & $53.80(16.54)$ & 0.216 \\
\hline Contralateral stimulation & $47.97(18.11)$ & $49.50(16.53)$ & 0.394 \\
\hline \multicolumn{4}{|l|}{ R2 area, asymptomatic side (mVms) } \\
\hline Ipsilateral stimulation & $2.40(1.13)$ & $2.26(0.86)$ & 0.544 \\
\hline Contralateral stimulation & $1.61(0.82)$ & $1.32(0.64)$ & 0.068 \\
\hline
\end{tabular}

${ }^{\mathrm{a} A l l}$ variables were normally distributed

prophylactic way in $\mathrm{CH}$, it may be difficult to measure nBR parameters during a $\mathrm{CH}$ attack.

The nBR was first studied in healthy subjects using a custom built concentric planar stimulating electrode allowing only the nociception specific A-delta fibers to be stimulated [3]. The nBR was further characterized in 104 healthy volunteers without any history of headache. Mean R2 onset latencies were $44.7 \mathrm{~ms}$ ipsilateral and $45.4 \mathrm{~ms}$ contralateral [6]. We are the first to present nBR reference values in $\mathrm{CH}$ patients and the effect of $\mathrm{O}_{2}$ on the nBR parameters. Consequently, it is not possible to make an accurate comparison with other nBR studies.
Our results of the $\mathrm{nBR}$ in $\mathrm{CH}$ and those from the literature raise some concerns about the applicability of the $\mathrm{BR}$ in $\mathrm{CH}$. We searched the literature for BR R2 parameters and found conflicting results with studies indicating no difference between $\mathrm{CH}$ patients and healthy controls [7], a decreased excitability in $\mathrm{CH}$ patients based on a lower R2 amplitude [8], or an increased excitability based on an increased R2 duration and amplitude [9].

If we combine these variable findings with our own results of the $\mathrm{nBR}$, we feel that the $\mathrm{nBR}$ may not be a suitable instrument for exploring the pathophysiology of $\mathrm{CH}$, although a previous BR study suggested otherwise 
[10]. We have to emphasize that most studies measured conventional non-nociceptive BRs, nevertheless without consistent results. We studied a fairly small homogeneous group of ten male $\mathrm{CH}$ patients. It is desirable to study a larger population with both male and female patients comparing $\mathrm{CH}$ patients in the active vs the remission phase. Also, the addition of healthy controls is necessary to compare values between groups in further studies.

We conclude that the $\mathrm{nBR}$ is not different between symptomatic and asymptomatic sides in patients during the active phase of $\mathrm{CH}$, outside of $\mathrm{CH}$ attacks, and that there is no measurable effect of $\mathrm{O}_{2}$ inhalation. Considering our observations with respect to the possible prophylactic action of SNS [5], it is questionable whether it will ever be possible to accurately measure the $\mathrm{nBR}$ during $\mathrm{CH}$ attacks.

\section{Abbreviations}

BR: Blink reflex; $\mathrm{CH}$ : Cluster headache; L/min: Liter/minute; nBR: Nociception specific blink reflex; $\mathrm{O}_{2}$ : Oxygen; pps: Pulse per second; R1: Early pontine response; R2: Late medullary response; SNS: Supraorbital nerve stimulation.

\section{Competing interests}

The authors declare that they have no competing interests.

\section{Authors' contributions}

$\mathrm{DH}$ drafted the study protocol, recruited patients, carried out the nBR measurements and helped to draft the manuscript. AP carried out the statistical analysis and drafted the manuscript. PK helped to draft the study protocol, recruited patients and helped to draft the manuscript. MH helped to draft the study protocol, advised on the execution of the nBR measurements, carried out the statistical analysis and helped to draft the manuscript. All authors read and approved the final manuscript.

\section{Acknowledgements}

The authors wish to thank J. Haan for his advice on the protocol of the study. This research did not receive a specific grant from any funding agency in the public, commercial or not-for-profit sectors. Materials were supplied by the Department of Neurology and Clinical Neurophysiology, Zuyderland Medical Center Heerlen, Sittard, The Netherlands.

\section{Author details}

${ }^{1}$ Department of Neurology and Clinical Neurophysiology, Zuyderland Medical Center Heerlen, P.O. Box 5500, 6130 MB Sittard, The Netherlands. ${ }^{2}$ Current address: Department of Neurology, Mariaziekenhuis Noord-Limburg, Overpelt, Belgium. ${ }^{3}$ Current address: Faculty of Health, Medicine and Life Sciences, Maastricht University, Maastricht, The Netherlands.

Received: 25 October 2015 Accepted: 2 February 2016

Published online: 12 February 2016

\section{References}

1. May A, Leone M, Áfra J, Linde M, Sándor PS, Evers S, Goadsby PJ, EFNS Task Force (2006) EFNS guidelines on the treatment of cluster headache and other trigeminal-autonomic cephalalgias. Eur J Neurol 13:1066-1077

2. Akerman S, Holland PR, Lasalandra MP, Goadsby PJ (2009) Oxygen inhibits neuronal activation in the trigeminocervical complex after stimulation of trigeminal autonomic reflex, but not during direct dural activation of trigeminal afferents. Headache 49:1131-1143

3. Kaube H, Katsarava Z, Käufer T, Diener HC, Ellrich J (2000) A new method to increase nociception specificity of the human blink reflex. Clin Neurophysiol 111:413-416

4. Aramideh M, Ongerboer De Visser BW, Koelman JHTM, Majoie CBL, Holstege $\mathrm{G}$ (1997) The late blink reflex response abnormality due to lesion of the lateral tegmental field. Brain 120:1685-1692
5. Haane DYP, Koehler PJ (2014) Nociception specific supraorbital nerve stimulation may prevent cluster headache attacks: Serendipity in a blink reflex study. Cephalalgia 34:920-926

6. Katsarava Z, Ellrich J, Diener HC, Kaube H (2002) Optimized stimulation and recording parameters of human 'nociception specific' blink reflex recordings. Clin Neurophysiol 113:1932-1936

7. Lozza A, Schoenen J, Delwaide PJ (1997) Inhibition of the blink reflex R2 component after supraorbital and index finger stimulations is reduced in cluster headache: An indication for both segmental and suprasegmental dysfunction? Pain 71:81-88

8. Raudino F (1990) The blink reflex in cluster headache. Headache 30:584-585

9. Formisano R, Cerbo R, Ricci M, Agostino R, Cesarino F, Cruccu G, Agnoli A (1987) Blink reflex in cluster headache. Cephalalgia 7:353-354

10. Van Vliet JA, Vein AA, Le Cessie S, Ferrari MD, Van Dijk JG, Dutch RUSSH research group (2002) Reproducibility and feasibility of neurophysiological assessment of the sensory trigeminal system for future application to paroxysmal headaches. Cephalalgia 22:474-481

\section{Submit your manuscript to a SpringerOpen ${ }^{\circ}$ journal and benefit from:}

- Convenient online submission

- Rigorous peer review

- Immediate publication on acceptance

- Open access: articles freely available online

- High visibility within the field

- Retaining the copyright to your article

Submit your next manuscript at $\boldsymbol{s p r i n g e r o p e n . c o m ~}$ 